\title{
Systemic vasculitis complicating infantile autoimmune enteropathy
}

\author{
H R Jenkins, F Jewkes, G M Vujanic
}

\begin{abstract}
An infant with unexplained sideroblastic anaemia and severe protracted diarrhoea due to autoimmune gut disease is reported. Despite treatment with an exclusion diet and immunosuppressive agents she developed a severe systemic vasculitic illness. Intensive treatment with plasmaphoresis resulted in resolution of the vasculitic process but the child subsequently died after overwhelming septicaemia.

(Arch Dis Child 1994; 71: 534-535)
\end{abstract}

Protracted diarrhoea of infancy is usually defined as the passage of four or more loose stools for longer than two weeks with loss of body weight, and is a rare and heterogeneous group of disorders in which the diagnosis can be identified in approximately $70 \%$ of cases. ${ }^{1}$

Autoimmune enteropathy is a recently described cause of protracted diarrhoea in young children and is characterised by severe gut damage, associated with the presence of circulating antienterocyte antibodies. ${ }^{2}$ Other organ specific autoimmune disease may also be present and treatment is based on the use of immunosuppressive agents such as prednisolone, azathioprine, and cyclosporin. ${ }^{23}$ The course of the disease is typically severe, often refractory to treatment and is potentially fatal. We describe an infant with severe autoimmune enteropathy, autoimmune liver disease, and idiopathic sideroblastic anaemia who, despite immunosuppressive treatment, developed a systemic vasculitic illness requiring intensive support for several weeks. Treatment with plasmaphoresis resulted in resolution of the vasculitic process but the child subsequently died of overwhelming septicaemia.

\section{Case report}

A girl was born to a primiparous woman at 39 weeks' gestation by emergency lower caesarean section because of fetal distress. Her birth weight was $2200 \mathrm{~g}$, she was formula fed, and there were no immediate problems. During the neonatal period, however, she suffered intermittent vomiting and loose stools. Poor weight gain became a significant problem at 3 months of age and initial investigations revealed a normal barium meal and follow through, haemoglobin concentration of $77 \mathrm{~g} / 1$ (having been $160 \mathrm{~g} / \mathrm{l}$ at 1 month of age) with no evidence of haemolysis, normal white cell count and platelets, bilirubin of $31 \mu \mathrm{mol} / 1$ (unconjugated), aspartate aminotransferase of
$85 U / 1$ (raised) and $\gamma$-glutamyltransferase of $484 \mathrm{U} / 1$ (raised). Clotting studies, full immunological studies, serum ferritin, creatinine and electrolytes, sweat test ultrasound of abdomen and head, computed tomography of the head, and cerebrospinal fluid examination were all normal.

She received a blood transfusion at 3 months of age and over the next three months she required three further transfusions because of a falling haemoglobin. A bone marrow examination showed sideroblastic anaemia with $20 \%$ ringed sideroblasts.

A jejunal biopsy specimen revealed severe hypoplastic villous atrophy with an acute and chronic inflammatory cell infiltrate and a liver biopsy specimen showed a picture of neonatal hepatitis, consistent with autoimmune hepatitis, with normal pancreatic function testing (after duodenal intubation and intravenous cholecystokinin/secretin). A full autoantibody screen showed positive antienterocyte antibodies but no other organ or non-organ specific autoantibodies.

She received intravenous feeding for three months and began to tolerate oral comminuted chicken (Chix; Cow and Gate) with good weight gain. Prednisolone was given intravenously for two weeks and this was continued as an oral dose $(2 \mathrm{mg} / \mathrm{kg} /$ day $)$ after which the diarrhoea improved. She continued to gain weight without parenteral nutrition and her liver function tests become normal, but the sideroblastic anaemia remained unexplained and was resistant to a three month course of pyridoxine, requiring monthly blood transfusion.

After a period of wellbeing and obvious improvement she developed a viral upper respiratory tract infection followed by a sudden collapse three days later with diarrhoea, vomiting, and the appearance of a widespread vasculitic skin rash. Her condition deteriorated rapidly requiring ventilation for respiratory and circulatory difficulties, in addition to increasing renal and liver failure. Antinuclear cytoplasmic antibody and all cultures (for bacteria, virus, and fungi) were negative. A diagnosis of widespread autoimmune disease with severe systemic vasculitis was made and her condition remained critical, despite the use of large doses of inotropic drugs, broad spectrum antibiotics, and antifungal treatment. She also received daily immunoglobulin and plasma infusion and regular blood transfusions. Haemofiltration was started because of increasing fluid overload due to renal failure and, after seven days of intensive support, double volume plasma exchange was instituted daily over five hours, using the haemofiltration circuit. This resulted in a gradual improvement 
in her condition and disappearance of the widespread vasculitic rash and she began to pass more urine. After lengthy consultation, cyclophosphamide was started and plasmaphoresis was reduced to alternate days. Despite repeated negative cultures, the possibility of sepsis was suspected and broad spectrum antibiotic treatment was given with teicoplanin, metronidazole, and amphotericin. There was an improvement in all parameters over a seven day period but she then rapidly became hypotensive and, despite vigorous attempts of resuscitation and careful consultation with the parents, support was withdrawn and she died peacefully in her parents' arms.

An immediate postmortem examination was carried out and revealed hepatic necrosis and steatosis, adrenal haemorrhages, renal microabscesses, and evidence of generalised septicaemia. Enterobacter cloacae, resistant to the antibiotics that she was given, was isolated from antemortem and postmortem cultures of blood, spleen, lung, and kidney. Histology of her small bowel was unremarkable with normal villi and no inflammatory cells, and was thus dramatically improved from previous appearances. The immediate cause of death was thought to be Gram negative septicaemia, presumably secondary to immunosuppressive treatment given for systemic vasculitis.

\section{Discussion}

Although autoimmune enteropathy is now a well described cause of protracted diarrhoea in young children, this is, to our knowledge, the first report of the development of widespread vasculitis associated with this condition. The associated sideroblastic anaemia has not previously been described and thus remains unexplained in our child. Immunosuppressive treatment with prednisolone did initially improve the child's condition but she suffered rapid deterioration which was resistant to further doses of prednisolone. It is possible that the severe enteropathy had already improved before plasmaphoresis as a consequence of her previous treatment with steroids and hypoallergenic feeds, or indeed that the autoimmune gut disease had already burnt itself out before the development of the widespread vasculitic illness. We were struck, however, by the dramatic improvement in her condition after plasmaphoresis and suggest that, in similar situations in the future, plasmaphoresis should be considered at an early stage as the improvement in the jejunal biopsy specimen was startling. It is tragic that she died from a sudden overwhelming septicaemia, after such an obvious improvement in her vasculitic illness.

We describe this case to highlight the association of autoimmune enteropathy with sideroblastic anaemia, the cause of which is unknown, and to report that plasmophoresis may be useful in the rare complication of systemic vasculitis after autoimmune enteropathy.

We would like to thank Marion Malone, Mike Dillon, Peter Milla, and Rita Marakian for their advice with this case, and Miss Leah Gallivan for typing the manuscript.

1 Larcher VF, Shepherd R, Francis DEM, Harris JT. Protracted diarrhoea of infancy. Analysis of 82 cases with Protracted diarrhoea of infancy. Analysis of 82 cases with particular references to diagn
Dis Child 1977; 52: 599-605.

2 Mirakian R, Richardson A, Milla PJ, et al. Protracted diarrhoea of infancy: evidence in support of an auto-immune variant. $B M \mathcal{F}$ 1986; 293: 1132-6.

3 Sanderson IR, Phillips AD, Spencer J, Walker-Smith JA. Response of auto-immune enteropathy to cyclosporin A therapy. Gut 1991; 32: 1421-5. 